\title{
Inequities in maternal health services utilization in Ethiopia 2000-2016: magnitude, trends, and determinants
}

\author{
Emebet Gebre ${ }^{1 *}$, Alemayehu Worku² and Fawole Bukola ${ }^{1}$
}

\begin{abstract}
Background: Inequities in maternal health services utilization constitute a major challenge in maternal mortality reduction in Ethiopia. We sought to assess magnitude, trends, and determinants of inequities in maternal health services utilization in Ethiopia from 2000 to 2016.

Methods: The study utilized data from the 2000 and 2016 Ethiopia Demographic and Health Surveys, which were done based on a cross sectional survey design. The wealth-related inequities were assessed by concentration curve and horizontal inequity indices. Trends in inequities were assessed by comparing the concentration indices of maternal health services utilization variables between the 2000 and 2016 surveys using Wagstaff two groups concentration indices comparison method. Finally, the inequities were decomposed into its contributing factors using Wagstaff method of analysis.

Results: Wealth-related inequities were significantly high in 2016: with horizontal inequities indices and residual regression error of antenatal care, skilled birth attendance, and postnatal care service utilization ( -0.09 and -0.01$)$, $(-0.06$ and 0.01$)$, and $(-0.11$ and 0.0001$)$, respectively. These indices increased significantly in 2016 when it is compared with the 2000 indices' with the respective concentration indices difference of $-0.05,0.05$, and -0.07 . The related all $p$-values were $<0.0001$. The main determinants of inequities were low-economic status, illiteracy, rural residence, no occupation, and fewer accesses to mass media.

Conclusions: In Ethiopia, maternal health services utilization inequities were significantly high and increased in 2016 compared to 2000. Women who are poor, rural resident, uneducated, unemployed, and fewer mass media exposed are the most disadvantaged. Targeting maternal health interventions for the underserved women is essential to reduce maternal mortality in the country.
\end{abstract}

Keywords: Determinants, Inequities, Maternal health services, Trend, Utilization

\section{Plain English summary}

Unfair difference among population groups (inequities) in maternal health services utilization is one of the challenges in reduction of maternal mortality in Ethiopia. The aims of the study were to assess magnitude, trends, and determinants of inequities in maternal health services utilization in Ethiopia from 2000 to 2016. Maternal health services utilization inequities were significantly high and increased in 2016 compared to 2000 with low-economic status,

\footnotetext{
* Correspondence: emuye2010@gmail.com

${ }^{1}$ Department of Obstetrics and Gynaecology, College of Medicine, Pan Africa University Life and Earth Sciences Institute, University of Ibadan, Ibadan, Nigeria

Full list of author information is available at the end of the article
}

illiteracy, rural residence, no occupation, and lack of access to mass media being the main determinants. In Ethiopia, the rich women were more likely to utilize maternal health services than the poor. The most disadvantaged women were the poor, rural resident, uneducated, unemployed, and with fewer/no mass media access. Targeting maternal health interventions for the underserved women is essential to reduce maternal mortality in the country.

\section{Background}

Worldwide 800 women die each day from childbirth and pregnancy related complications [1]. Out of the total worldwide maternal deaths, $99 \%$ occurred in developing 
countries and sub-Saharan Africa bears the largest share (66\%). Relative to other developing countries, maternal mortality ${ }^{1}$ remains high in Ethiopia with a maternal mortality ratio of 412 per 100, 000 live birth [2].

Inequities $^{2}$ in maternal health services utilization in Ethiopia is one of the highest in the world [3], which is also one of the contributing factors for high maternal mortality in the country [4], although developing an equitable standard of health services for all segments of the population is one of the general health policies of Ethiopian government [5].

Maternal and new-born deaths can be prevented by implementing key.

essential services for mothers including antenatal care (having at least four or more antenatal care visits during pregnancy), skilled attendance at birth, and postnatal care $[6,7]$.

In many developing countries including Ethiopia, access to these lifesaving services is limited among key population groups due to low socioeconomic status [814] and remains as one of the challenges in maternal mortality reduction [15]. Thus understanding the current status, trend, and contributing factors of inequities in maternal health service utilization is crucial to achieve the Sustainable Development Goal (SDG) that aims to reduce maternal mortality to 70 per 100,000 live births by 2030 [16]. Inequities in maternal health services utilization has been reported in Ethiopia [9-14], although the approaches were divergent. Some of these studies were based on a subset of population that were on the two extreme poles of wealth continuum between the richest and the poorest segment of the population [17]. This approach excludes the subset of population between these two poles and thus does not provide a full picture of the effect of wealth index on maternal health services utilisation [18]. Utilisation of health services and health inequalities do not depend only on a wealth factor but also other need factors that increase individual's attendance of health care can confound the effect of wealth index on maternal health services utilisation. The effect of such need factors was not taken into account in some studies [11]. Studies that include all the population segments irrespective of wealth status of individuals as well as those that account for the effect of potential confounders (need factors) are required to fully understand health inequalities and its determinants $[18$, 19]. Measurement of inequity and its trends as data becomes available is important to monitor regularly the country progress towards achieving sustainable development goal (achieving universal health coverage for essential maternal health interventions for all by 2030) [16]. Thus, the aims of this study were to assess the magnitude, trends, and determinants of inequities in maternal health services utilization in Ethiopia using nationally representative data that became available recently. Such that, the country's progress towards achieving sustainable development goals can be tracked.

\section{Methods \\ Data}

We used nationally representative Ethiopia Demographic and Health Surveys (EDHS) data from 2000 to 2016 publicly available via Measure DHS. EDHS are nationally representative household surveys conducted at 5-yearly intervals with a strong focus on indicators of maternal and child health, reproductive health, fertility, nutrition, mortality, and self-reported health behaviors among adults. Demographic and health surveys are considered as providing an important source of monitoring population health indicators and vital statistics in middle and low-income countries and known by its design, which are highly comparable among different settings and over time $[20,21]$. All EDHS were conducted using a similar approach in sample design, sample selection, and survey methodology (each round survey methodology is stated in the respective reports) and ethically approved [2, 22-25].

\section{Socioeconomic rank}

We used the 2016 EDHS wealth index (WI) variable as a living standard measure for the 2016 data. Since WI variable was not available in the 2000 EDHS data, we constructed the WI variable using the Principal Component Analysis (PCA) as a measure of socioeconomic status used in DHS reports of many countries [26]. The variables included in the PCA were durable assets ownership: radio, car, refrigerator, television, motorcycle, and bicycle; housing characteristics: number of rooms for sleeping and building material (wall, floor and roof); and access to utilities and infrastructure: electricity supply, source of drinking water, and sanitation facility.

\section{Measures}

We assessed inequities in antenatal care (ANC), skilled birth attendant (SBA) and postnatal care (PNC) services utilization - the most prioritized maternal health interventions in securing the continuum of care for maternal and child health [27]. ANC was calculated as the number of women who had at least four or more ANC visits for the last birth in five years preceding the survey. Similarly, SBA was defined as the number of women who were assisted by a skilled health provider (Doctor or Nurse or Midwife for the 2000 EDHS data and Doctor or Nurse or Midwife or Health Officer or Health Extension Worker for 2016 EDHS data) for the last live birth in five years preceding the survey. PNC was computed as the number of women who had postnatal check-up within two days after delivery for the last birth in five 
years preceding the survey except for the 2016 surveys (within two years preceding the survey).

\section{Explanatory variables descriptions}

The explanatory variables were women's wealth status, residence (urban or rural), education, occupation, mass media exposure, respondent's current age and current marital status.

\section{Data analysis}

\section{Measuring inequity}

Inequities in Maternal Health Services (MHS) utilization were measured by Horizontal Inequity Indices (HII) and concentration curve following Wagstaff method of analysis [28]. Since age and four or more birth order of a woman may affect wealth status of a woman [29] and may correlate with MHS utilization [30]; inequities were determined after standardizing MHS utilization variables using indirect approach. To indirectly standardize each of the health variables, a nonlinear method of estimation using logit model was used.

$$
y_{i}=\alpha+\sum_{j} \beta_{j} X_{j i}+\sum_{k} y_{k} z_{k i}+\varepsilon_{i}
$$

where $\alpha, \beta$, and $\gamma$ are parameter vectors; $y_{i}$ is MHS utilization; $x$ is the need factors (confounding variables) such as women age and four or more birth order; $z$ is non-need factors (non-confounding variables) to control or estimate the partial correlation with confounding variables. The non-need factors were education status, rural resident, low-economy status, current marital status, occupation, mass media exposure (a predictor of MHS utilization [31]). $\varepsilon$ is an error term. For our case we used Logit model.

The indirectly standardized MHS utilization estimate is given by the difference between actual MHS utilization $\mathrm{y}_{\mathrm{i}}$ and the expected utilization (utilization expected only from need factors), plus the total sample mean of MHS utilization $\bar{y}$ [32].

$$
\hat{y}_{i}^{I S}=y_{i}-\hat{y}_{i}^{X}+\bar{y}
$$

In this study, concentration curve plots the cumulative percentage of the MHS utilization variable (on the vertical axis) against the cumulative percentage of the population ranked by wealth index starting with the poorest (left) and ending with the better-off (right) on the horizontal axis $[33,34]$.

Concentration Index $(\mathrm{CI})$ is calculated from concentration curve as it is defined as twice the area between the concentration curve and the line of equality [34]. Using eq. 3 [35].

$$
C=\frac{2}{n \cdot \mu} \sum_{i=1}^{n} y_{i} R_{i}-1,
$$

where $\mathrm{C}$ is the concentration index, $\mu$ is the mean of $y_{i}$ (MHS utilization), $R_{i}$ is the fractional rank of the $i$ th individual in the income distribution.

Concentration Index can also be calculated simply by following the "convenient regression approach". This kind of calculation gives both the estimates and also the standard errors to produce statistical inferences.

It can be written as follows:

$$
2 \sigma_{r}^{2}\left(y_{i} / \mu\right)=\alpha+\beta r_{i}+\varepsilon_{i}
$$

Where, $\beta$ is an estimated concentration index, $\sigma_{r}^{2}$ is the variance of the rank $(r)$, the other variables are as defined in eq. (3), and is the stochastic error term.

Then, we got MHS Horizontal Inequity Indices (HII) and residual by calculating the concentration indices using the need standardized MHS utilization variables [36]. When there is no inequality, the horizontal inequity index will be zero and the concentration curve lies on the diagonal line starting from the origin (line of equality); which means all individuals, regardless of their economic status have the same value of the health variable. If there is inequity, CI will be negative or positive indicating that the MHS utilization variable is more concentrated among poor or better-off group of people and concentration curve lies above or below the line of equality and the HII value lies between -1 and 1 . The further concentration curve lays from the line of equality, the greater the degree of inequality in MHS utilization across income groups. Similarly, the higher the absolute CI value is the greater the inequity in MHS utilization [28, 32].

Trends in inequities of MHS utilization were investigated using Wagstaff two groups (2000 and 2016 EDHS) concentration indices comparison method via the "CONINDEX" STATA commands [37].

\section{Decomposition}

To examine the socioeconomic determinants of inequities in MHS utilization, decomposition of the indirectly standardized concentration indices were done following a method of analysis proposed by Wagstaff et al. [28, 36].

A linear regression model joining our variables of interest (MHS utilization), $\mathrm{y}$, to a set of $k$ determining factors, $\mathrm{x}_{\mathrm{k}}$ :

$$
y_{i}=\alpha+\sum_{k} \beta_{k} x_{k i}+\varepsilon_{i}
$$

Where, $\beta_{\mathrm{k}}$ is coefficient of health determinants, $\varepsilon_{\mathrm{i}}$ is an error term.

Given the association between $y i$ and $x_{k i}$ in Eq. (5), the concentration index for $y, C I$, can be written as: 


$$
c=\sum_{k}\left(\frac{\beta_{k} \bar{x}_{k}}{\mu}\right) c_{k}+\frac{G C_{\varepsilon}}{\mu}=c_{\hat{y}}+\frac{G C_{\varepsilon}}{\mu},
$$

where, $\beta_{\mathrm{k}}$ is the regression coefficient of variable $x_{k}, \mu$ is the mean of $\mathrm{y}, \bar{x}_{k}$ is the mean of variable $\mathrm{x}_{\mathrm{k}}, \mathrm{C}_{\mathrm{k}}$ is $\mathrm{CI}$ of variable $x_{k}, \mu$ is the mean of MHS utilization variable and GC is generalized CI for the an error term $(\varepsilon)$; which can be computed as a residual and can be defined as:

$$
G C_{\varepsilon}=\frac{2}{n} \sum_{i=1}^{n} \varepsilon_{i} R_{1},
$$

The CI in eq. (6) shows that it is made up of two components. The first component is deterministic (explained) which is equal to weighted sum of concentration indices of $k$ regressors, where the weight or "share" for $\mathrm{x}_{\mathrm{k}}$ is simply the elasticity of $y$ with respect to $x_{k}$ when evaluated at the sample mean. An elasticity is a unit-free measure of (partial) association, i.e. the percentage change in the dependent variable (MHS utilization in this case) associated with a percentage change in the explanatory variable of $\mathrm{y}$ with respect to each $\left.\mathrm{x}_{\mathrm{k}}, \frac{\beta_{k} \bar{x}_{k}}{\mu}\right)$. But, the second component is the residual, which reflects the inequality in health service utilization that cannot be explained by systematic variation in the $x_{k}$ across socioeconomic groups [28, 32].

The data were processed and analyzed using EXCEL and STATA version 13.0 [34]. In addition, ADePT software version 6 was used to analyse the socioeconomic inequities and decomposition [38]. We incorporated EDHS data unequal sampling weight and household clustering effect in the analyses [39]. Sample-weighted data were used for all of the analyses to adjust for the under-sampling, the over-sampling, and the response rates differences in different regions.

\section{Results}

\section{Demographic and socio-economic characteristics of respondents}

The study participants in Ethiopia DHS were taken from the nine geographic regions and two administrative states (Addis Ababa City administration and Dire Dawa city council). The proportion of population that resides in rural area in each of these nine regions ranges from $50 \%$ in Harari region to $90 \%$ in Southern Nation Nationality People Region (SNNPR). Majority of the respondents were 15-19 years old, rural resident, illiterate, married, and from Oromya region both in 2000 and 2016 (Table 1).

\section{Inequities in maternal health services (MHS) utilization in Ethiopia in 2016}

Inequities in MHS utilization were assessed by concentration curve, concentration indices, and horizontal inequity indices.
Table 1 Demographic and socio-economic characteristics of

\begin{tabular}{|c|c|c|c|}
\hline \multicolumn{2}{|l|}{ Characteristics } & \multicolumn{2}{|l|}{ Year } \\
\hline & & $\begin{array}{l}2000 \\
N(\%)\end{array}$ & $\begin{array}{l}2016 \\
N(\%)\end{array}$ \\
\hline \multirow[t]{7}{*}{ Age (years) } & $15-19$ & $3584(23.3)$ & $3498(22.3)$ \\
\hline & $20-24$ & $2844(18.5)$ & $2903(18.5)$ \\
\hline & $25-29$ & $2716(17.7)$ & $2845(18.1)$ \\
\hline & $30-34$ & $1902(12.4)$ & $2241(14.3)$ \\
\hline & $35-39$ & $1762(11.5)$ & $1917(12.2)$ \\
\hline & $40-44$ & $1324(8.6)$ & 1302(8.3) \\
\hline & $45-49$ & $1235(8.0)$ & $977(6.2)$ \\
\hline \multirow[t]{2}{*}{ Residence } & Urban & 4543(29.6) & $5348(34.1)$ \\
\hline & Rural & $10,824(70.4)$ & $10,335(65.9)$ \\
\hline \multirow[t]{4}{*}{ Education Level } & No education & $10,586(68.9)$ & $7033(44.8)$ \\
\hline & Primary & $2530(16.5)$ & $5213(33.2)$ \\
\hline & Secondary & 2092(13.6) & $2238(14.3)$ \\
\hline & Higher & 159(1.0) & $1199(7.7)$ \\
\hline \multirow[t]{5}{*}{ Wealth index } & Poorest & $2885(21.1)$ & $3894(24.8)$ \\
\hline & Poorer & $806(5.9)$ & 2046(13.1) \\
\hline & Medium & 2731(19.9) & $2002(12.8)$ \\
\hline & Rich & 2553(18.7) & $2042(13.0)$ \\
\hline & Richest & $4684(34.3)$ & $5699(36.3)$ \\
\hline \multirow[t]{6}{*}{ Marital status } & Never married & $3979(25.9)$ & $4278(27.3)$ \\
\hline & Married & $9203(59.9)$ & $9602(61.2)$ \\
\hline & Living together & $177(1.2)$ & $222(1.4)$ \\
\hline & Widowed & $657(4.3)$ & $451(2.9)$ \\
\hline & Divorced & $926(6.0)$ & 878(5.6) \\
\hline & Not living together & $425(2.8)$ & $252(1.6)$ \\
\hline \multirow[t]{11}{*}{ Region } & Tigray & $1306(8.5)$ & $1682(10.7)$ \\
\hline & Affar & $858(6.6)$ & $1128(7.2)$ \\
\hline & Amhara & 1909(12.4) & $1719(11.0)$ \\
\hline & Oromiya & 2578(16.8) & $1892(12.1)$ \\
\hline & Somali & $844(5.5)$ & $1391(8.9)$ \\
\hline & Benshal-gumz & $992(6.5)$ & $1126(7.2)$ \\
\hline & SNNPR & $2028(13.2)$ & 1849 (11.8) \\
\hline & Gambela & $876(5.7)$ & 1035 (6.6) \\
\hline & Harari & $908(5.9)$ & $906(5.8)$ \\
\hline & Addis & $2015(13.11)$ & 1824(11.6) \\
\hline & Dire Dawa & $1053(6.9)$ & $1131(7.2)$ \\
\hline
\end{tabular}
respondents in Ethiopia by survey year (2000 \& 2016)

Figure 1 shows concentration curves of MHS utilization variables. All of the concentration curves lie above the line of equity, indicating that the non-utilization is concentrated among the poorest than the better-off women.

The horizontal inequity indices (HII) and their residual regression errors of ANC, SBA, and PNC services utilization were found as ( $\mathrm{HII}$ and residual regression 

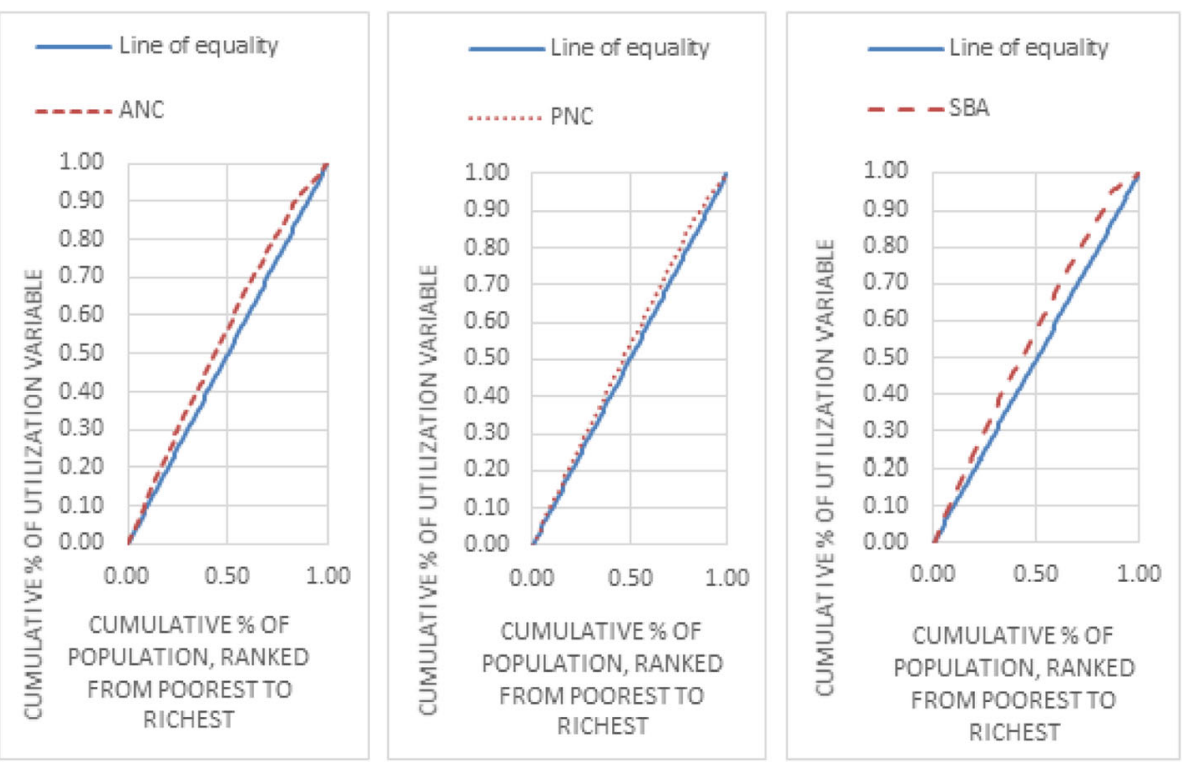

Fig. 1 Maternal health services utilization concentration curves in Ethiopia in 2016

errors) $(-0.10$ and 0.01$),(-0.11$ and 0.0001$)$, and $(-0.06$ and -0.01), respectively in 2016. Similarly, the horizontal inequity indices show considerable inequity with MHS non- utilization concentrated among the poor women than those who were better-off.

Trends in MHS utilization inequities by wealth quintile in Ethiopia from 2000 to 2016

The likelihood of better-off than poor women in the utilization of ANC, SBA, and PNC check-up services inequities- increased significantly in 2016 related to 2016 (Table 2, Additional files 1, 2, and 3).

\section{Decomposition of the concentration indices}

We decomposed the determinants of MHS utilization inequities in Ethiopia using data from the 2016 EDHS data.

The dominant determinants of inequities in ANC service utilization (three or fewer ANC visits) inequity were low wealth status (37\%), followed by rural residence (32\%) and being illiterate (17\%). Additionally, less than twice a week mass media exposure (3\%) was found to be a significant determinant of inequity (Table 3, Fig. 2).

The leading determinants of inequities in SBA service utilization were being a rural resident (44\%) followed by low wealth status (34\%) and illiteracy (17\%). Having less than twice a week mass media exposure (3\%) was also found as a significant determinant of the inequity (Table 3, Fig. 2).

The major determinants of inequity in PNC check-up were illiteracy (35\%) followed by low wealth status (32\%) and rural residence (15\%). In addition, occupation (2\%) was found as a significant determinant of the inequity (Table 3, Fig. 2).

The decomposition residual regression errors of inequities in ANC, PNC, and SBA services utilization were found as $-0.01,-0.01$, and 0.0001 , respectively, which are very small and indicate factors included in the decomposition analysis explained most of the inequities (Table 3).

Generally, considerable inequity exists among the study population poor, rural resident, illiterate, unemployed, and women with no/fewer mass media exposure.

\section{Discussion}

We found that considerable inequities exist among the study population and non-utilization of MHS is concentrated among the disadvantage women. In Ethiopia, poor women were less likely than better-off in utilizing ANC,

Table 2 The trends of MHS utilization inequities in Ethiopia from 2000 to 2016

\begin{tabular}{|c|c|c|c|c|c|c|c|}
\hline & & & & & Z-test & & \\
\hline & & $\mathrm{Cl}$ & SE & $p$-value & Difference & SE & $p$-value \\
\hline \multirow[t]{3}{*}{ ANC } & 2000 & -0.04 & 0.01 & 0.0001 & & & \\
\hline & 2016 & -0.09 & 0.01 & 0.0001 & & & \\
\hline & & & & & -0.0518 & 0.0099 & $<0.0001$ \\
\hline \multirow[t]{3}{*}{ PNC } & 2000 & -0.0042 & 0.0015 & 0.0060 & & & \\
\hline & 2016 & -0.0540 & 0.0051 & $<0.0001$ & & & \\
\hline & & & & & -0.0499 & 0.0053 & $<0.0001$ \\
\hline \multirow[t]{3}{*}{ SBA } & 2000 & -0.03 & 0.00 & $<0.0001$ & & & \\
\hline & 2016 & -0.10 & 0.01 & $<0.0001$ & & & \\
\hline & & & & & -0.07 & 0.01 & $<0.0001$ \\
\hline
\end{tabular}


Table 3 Decomposition of MHS utilization concentration indices (Ethiopia, 2016)

\begin{tabular}{|c|c|c|c|c|c|}
\hline & Coefficient & Elasiticity & Covariates $\mathrm{Cl}$ & Absolute Contribution & Percentage Contribution \\
\hline \multicolumn{6}{|l|}{ ANC } \\
\hline \multicolumn{6}{|l|}{ Standardize variables } \\
\hline Respondent's current age & -0.003 & -0.116 & 0.001 & 0.000 & 0 \\
\hline Birth order $4^{+}$ & 0.057 & 0.045 & -0.080 & -0.004 & 4 \\
\hline \multicolumn{6}{|l|}{ Control variables } \\
\hline Low wealth status & 0.091 & 0.062 & -0.564 & -0.035 & 37 \\
\hline Rural & 0.235 & 0.293 & -0.104 & -0.030 & 32 \\
\hline Illiterate & 0.117 & 0.112 & -0.144 & -0.016 & 17 \\
\hline Occupation & -0.019 & -0.015 & 0.067 & -0.001 & 1 \\
\hline Mass media exposure & 0.239 & 0.445 & -0.006 & -0.003 & 3 \\
\hline Current marital status & 0.007 & 0.013 & -0.008 & 0.000 & 0 \\
\hline Residual & & & & & -0.01 \\
\hline \multicolumn{6}{|l|}{ PNC } \\
\hline \multicolumn{6}{|l|}{ Standardizing variables } \\
\hline Respondent's current age & -0.001 & -0.034 & 0.001 & 0.000 & 0 \\
\hline Birth order $4^{+}$ & 0.044 & 0.025 & -0.080 & -0.002 & 4 \\
\hline \multicolumn{6}{|l|}{ Control variables } \\
\hline Wealth status (low) & 0.055 & 0.032 & -0.564 & -0.018 & 32 \\
\hline Illiterate & 0.079 & 0.058 & -0.144 & -0.008 & 15 \\
\hline Rural & 0.230 & 0.191 & -0.104 & -0.020 & 35 \\
\hline Mass media exposure & 0.012 & -0.005 & -0.006 & 0.000 & 0 \\
\hline Occupation & -0.026 & -0.014 & 0.067 & -0.001 & 2 \\
\hline Current marital status & -0.002 & -0.003 & -0.007 & 0.000 & 0 \\
\hline Residual & & & & & -0.01 \\
\hline \multicolumn{6}{|l|}{ SBA } \\
\hline \multicolumn{6}{|l|}{ Standardizing variables } \\
\hline Respondent's current age & -0.003 & -0.160 & 0.001 & 0.000 & 0 \\
\hline Birth order $4^{+}$ & 0.153 & 0.141 & -0.071 & -0.010 & 9 \\
\hline \multicolumn{6}{|l|}{ Control variables } \\
\hline Low wealth status & 0.094 & 0.071 & -0.532 & -0.038 & 34 \\
\hline Rural & 0.425 & 0.560 & -0.089 & -0.050 & 44 \\
\hline Illiterate & 0.149 & 0.148 & -0.132 & -0.020 & 17 \\
\hline Occupation & -0.014 & -0.010 & 0.066 & -0.001 & 1 \\
\hline Mass media exposure & 0.173 & 0.512 & -0.006 & -0.003 & 3 \\
\hline Current marital status & -0.001 & 0.000 & -0.005 & 0.000 & 0 \\
\hline Residual & & & & & 0.00 \\
\hline
\end{tabular}

SBA, and PNC services in 2016. This finding is similar to earlier studies [11, 17, 40-47].

Compared to the baseline, 2000 survey; the likelihood of ANC, SBA, and PNC services utilization was significantly higher among better-off than poor women in 2016. This finding is similar to previous reports $[9,17$, $41,48-50]$, but contradicts some former studies [47, 50]. This may be for PNC due to PNC service utilization increased significantly among better-off women but not among poor from 2000 to 2016, for SBA due to statistically insignificant change in utilization of SBA service coverage amongst both well off and poorest from 2000 to 2016 [22]; and generally due to the rapid population growth observed in the country from 2000 to 2017 [51], which may increase the health care demands of the people, a low health facility to population ratio, and it may be also due to low health workforce $(0.7$ health workers per population) in the country compared to the 


\section{Proportional contributions of determinants of MHS utilization inequities}

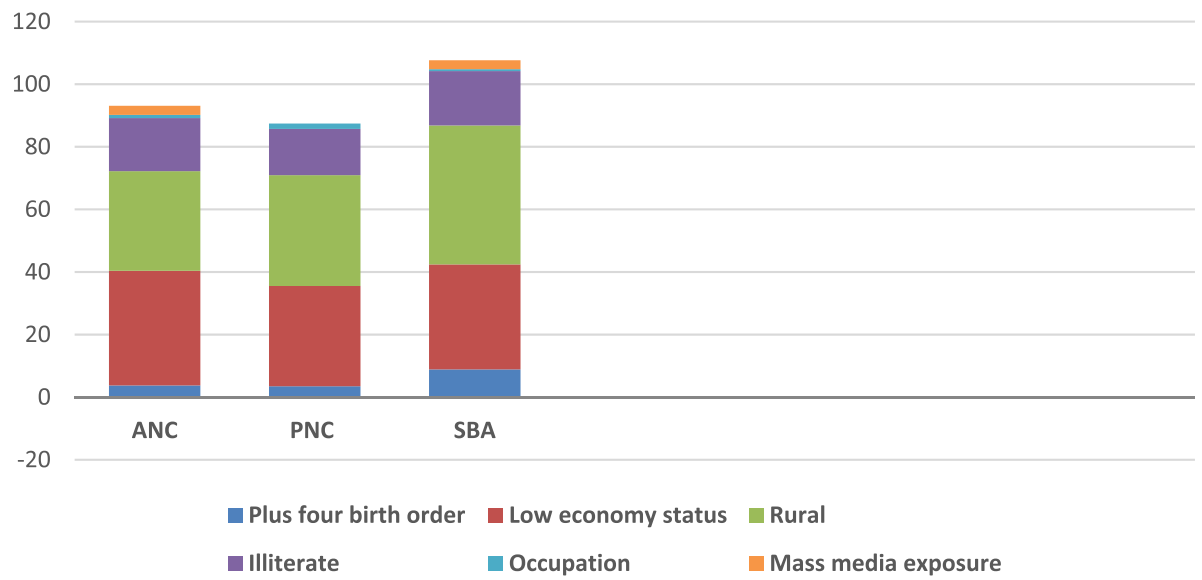

Fig. 2 Proportional contributions of determinants of MHS utilization inequities in Ethiopia in 2016

WHO recommendation (2.3 health workers per 1000 population) [52]. However, it should be noted that the computation of wealth indices for the two surveys is different with urban-rural differences taken into account in the 2016 survey.

Low economic status was found as a major determinant of inequities in MHS utilization. This may be poor women may not have money to cover payments for transport and either for the service or other expenses to bring and keep families at a health facility. This is similar to prior reports [41, 53-55].

Similarly, rural residence was found as a major determinant of inequities in all the studied MHS utilization. This finding is similar to some previous studies [11, 42, $44,55-57]$. Lack of access to health care services in rural areas due to inaccessibility of health facilities and professionals, lack of transportation services, and less access to infrastructures and services play a key role in the observed inequality. Thus, rural and remote areas are often underserved by health workers [52]. In addition, women in rural places are less educated and less autonomous than their urban counterparts to actively seek health cares.

Likewise, illiteracy is a major determinant of inequity in MHS utilization. Illiterate women are often unemployed, lack independent decision-making ability, and awareness about the importance of having MHS. A similar finding was reported from Vietnam [54].

Mass media exposure was found to be a significant determinant of inequities in MHS utilization. Women exposed to mass media at least twice a week were more likely to utilize MHS than those less exposed. This finding is similar to a report from India $[53,58]$. This may be because those more exposed to mass media may have good awareness about the importance of utilizing the services.
This study does have some limitations. The first limitation is recall bias because the responses were based on the mother's recall. The second limitation is related to asset indices. We identified that the wealthiest quintile inclined to reside in urban places; this indicates that the wealth inequalities may be related to rural/urban disparities. The third limitation is household asset based inequalities exclude inequalities related to age, ethnic group, or position in the household family structure. The fourth limitation is the absence of some need factors in EDHS for interventions may not be included and bias the horizontal inequity indices. In addition, there exist some variations in the definitions of SBA and PNC service utilization variables in terms of the type of health care provider and timings of service provision between the 2000 and 2016 EDHS. Thus, interpretation of findings in this study should consider such variations.

Despite these limitations, this study assessed the objectives using the recent and publicly available data for a relatively long-time period to see the general trends and magnitudes and contributing factors of inequities using the most recent EDHS data that are nationally representative and do have high response rates. The data were collected after high-quality interviewer training was given to the data collectors. The data were collected using a standardized data collection procedures across countries - to make its content consistent over time and comparable across populations [21]. The conclusions of this study were based on the concentration index results, which fulfil the minimum requirement of an inequality measurement rather than inequality ratio results - based on only the extreme quintiles of a population - to compare inequalities across different time periods $[18,19]$.

Generally, the study shows that the observed increment in the utilization of MHS is only among better off not among poor. 


\section{Conclusion}

Our findings show the presence of significantly high and increasing inequities in ANC, SBA, and PNC utilization. The poorer, illiterate, rural residents, not working, and mass media unexposed women (the majority in Ethiopia) were the disadvantaged segments in Ethiopia and health interventions should target them.

\section{Endnotes}

${ }^{1}$ Maternal mortality is defined as "the death of a woman while pregnant or within 42 days of termination of pregnancy, irrespective of the duration and site of the pregnancy, from any cause related to or aggravated by the pregnancy or its management, but not from the accidental or incidental cause [59]."

${ }^{2}$ Inequality in health care is differences in the health care utilization by clusters or person $[60,61]$. Whereas, equity in health cares: "is defined as equal access to available care for equal need, equal utilization for equal need, and equal quality of care for all."

\section{Additional files}

Additional file 1: Figure S1. ANC service utilization gap trend among poorest and richest population, in Ethiopia from 2000 to 2016. (DOCX $17 \mathrm{~kb}$ )

Additional file 2: Figure S2. SBA service utilization gap trend among poorest and richest population, in Ethiopia from 2000 to 2016. (DOCX $16 \mathrm{~kb}$ )

Additional file 3: Figure S3. PNC service utilization gap trend among poorest and richest population, in Ethiopia from 2000 to 2016. (DOCX $16 \mathrm{~kb}$ )

\section{Abbreviations}

ADePT: Automated DEC (Development Economics) Poverty Table; ANC: Antenatal Care; Cl: Concentration Index/Indices; CONINDEX: Concentration Index; CSA: Central Statistical Agency; EDHS: Ethiopia Demographic Health Survey/s; MHS: Maternal Health Services; PNC: Postnatal care; RCl: Relative Concentration Index/Indices; SBA: Skilled Birth Attendance; WHO: World Health Organization

\section{Acknowledgments}

We are grateful to Africa Union, PAN Africa University Life and Earth Science Institute for sponsoring this study and Obstetrics and Gynaecology Department, the University of Ibadan for running a program. We want to express our gratitude to Measure DHS and Ethiopia Central Statistical Agency for proving Ethiopia Demographic Health Surveys data. The professional support provided by Prof. Oladapo Olayemi, Prof. Ayodele Arowojolu, and Mr. Debebe Shaweno are very much acknowledged.

\section{Funding}

The study was sponsored by PAN Africa University Life and Earth Science Institute, University of Ibadan, Ibadan, Nigeria. The funding institute had not any role in collecting and analysing the data, interpreting of the results, writing the report and deciding to submit the manuscript for publication.

\section{Availability of data and materials}

The used EDHS data are publicly available via Measure DHS and Ethiopia Central Statistical Agency.

\section{Authors' contributions}

GE conceived and designed the study, performed the data analyses and writes up, drafted the manuscript and prepared it for publication. WA participated in designing the study and critically reviewing the manuscript for intellectual content. BF participated in designing the study and critically reviewing the manuscript for intellectual content. All authors read and approved the final manuscript.

\section{Ethics approval and consent to participate}

All EDHS data were collected after the study got an ethical clearance from at least one of the following institutes: Ethiopia Central Statistical Authority, Ethiopia Central Statistical Agency (CSA), Ethiopia Health and Nutrition Research Institute (EHNRI) Review Board, the National Research Ethics Review Committee (NRERC) at the Ministry of Science and Technology, the Institutional Review Board of ICF International, and the CDC. Consent was obtained from each study participant before conducting an interview. We obtained the data by submitting the data request to Measure DHS

\section{Consent for publication}

Not applicable.

\section{Competing interests}

The authors declare that they have no competing interest.

\section{Publisher's Note}

Springer Nature remains neutral with regard to jurisdictional claims in published maps and institutional affiliations.

\section{Author details}

'Department of Obstetrics and Gynaecology, College of Medicine, Pan Africa University Life and Earth Sciences Institute, University of Ibadan, Ibadan, Nigeria. ${ }^{2}$ School of Public Health, Addis Ababa University, Addis Ababa, Ethiopia.

Received: 4 April 2018 Accepted: 13 June 2018

Published online: 04 July 2018

\section{References}

1. WHO: Global strategy for Women's, Children's and Adolescents' health (2016-2030): data portal. 2017

2. Central Statistical Agency (CSA) [Ethiopia], ICF. Ethiopia Demographic and Health Survey 2016. Addis Ababa, Ethiopia, and Rockville, Maryland, USA: CSA and ICF; 2016.

3. Barros AJ, Ronsmans C, Axelson H, Loaiza E, Bertoldi AD, França GV, Bryce J, Boerma JT, Victora CG. Equity in maternal, newborn, and child health interventions in countdown to 2015: a retrospective review of survey data from 54 countries. Lancet. 2012;379(9822):1225-33.

4. Ruhago GM, Ngalesoni FN, Norheim OF. Addressing inequity to achieve the maternal and child health millennium development goals: looking beyond averages. BMC Public Health. 2012;12(1):1119.

5. FDRE: Health policy of the transitional government of Ethiopia in. Edited by $\mathrm{MOH} ; 1993$.

6. Korn EL, Graubard BI. Analysis of health surveys, vol. 323: John Wiley \& Sons; 2011.

7. Maternal health [https://www.unfpa.org/maternal-health]. Accessed 21 June 2017

8. Say L, Raine R. A systematic review of inequalities in the use of maternal health care in developing countries: examining the scale of the problem and the importance of context. Bull World Health Organ. 2007;85(10):812-9.

9. Tesfaye B, Mathewos T, Kebede M. Skilled delivery inequality in Ethiopia: to what extent are the poorest and uneducated mothers benefiting? Int J Equity Health. 2017;16(1):82.

10. Fekadu M, Regassa N. Skilled delivery care service utilization in Ethiopia: analysis of rural-urban differentials based on national demographic and health survey (DHS) data. Afr Health Sci. 2014;14(4):974-84.

11. Bobo FT, Yesuf EA, Woldie M. Inequities in utilization of reproductive and maternal health services in Ethiopia. Int J Equity Health. 2017;16(1):105.

12. Aliy J, Mariam DH. Determinants of equity in utilization of maternal health services in Butajira, Southern Ethiopia. Ethiopian Journal of health development. 2012;26(1):265-70.

13. Ambel AA, Andrews C, Bakilana AM, Foster EM, Khan Q, Wang H. Examining changes in maternal and child health inequalities in Ethiopia. Int J Equity Health. 2017;16(1):152.

14. Wagstaff A. Poverty and health sector inequalities. Int J Public Health. 2002; 80(2):97-105.

15. Ministry of Health Ethiopia, PMNCH, WHO, World Bank, AHPSR: Success factors for women's and children's health: Ethiopia. In. Switzerland; 2015 
16. Sustainable Development Goal 3: Ensure healthy lives and promote wellbeing for all at all ages [https://sustainabledevelopment.un.org/sdg3]. Accessed 5 Aug 2017.

17. Alam N, Hajizadeh M, Dumont A, Fournier P. Inequalities in maternal health care utilization in sub-Saharan African countries: a multiyear and multicountry analysis. PLoS One. 2015;10(4):e0120922.

18. Wagstaff $A$, Paci $P$, Van Doorslaer E. On the measurement of inequalities in health. Soc Sci Med. 1991;33(5):545-57.

19. Wong KL, Restrepo-Méndez MC, Barros AJ, Victora CG. Socioeconomic inequalities in skilled birth attendance and child stunting in selected low and middle income countries: wealth quintiles or deciles? PLoS One. 2017; 12(5):e0174823.

20. WHO. State of Inequality: Reproductive Maternal Newborn and Child Health Interactive Visualization of Health Data. Geneva: World Health Organization; 2015.

21. Corsi DJ, Neuman M, Finlay JE, Subramanian S. Demographic and health surveys: a profile. Int J Epidemiology. 2012;41(6):1602-13.

22. Central Statistical Agency [Ethiopia]: Ethiopia Mini Demographic and Health Survey 2014. In. Addis Ababa, Ethiopia; 2014

23. Central Statistical Agency [Ethiopia] and ICF International: Ethiopian Demograhpic Health Survey 2011. In. Addis Ababa, Ethiopia and Calverton, Maryland, USA: Central Statistical Agency and ICF International; 2012.

24. Central Statistical Authority [Ethiopia] and ORC Macro. Ethiopia Demographic and Health Survey 2000. Addis Ababa, Ethiopia and Calverton, Maryland, USA: Central Statistical Authority and ORC Macro; 2001.

25. Central Statistical Agency [Ethiopia] and ORC Macro. Ethiopia Demographic and Health survey 2005. Addis Ababa, Ethiopia and Calverton, Maryland, USA: Central Statistical Agency and ORC Macro; 2006

26. Vyas S, Kumaranayake L. Constructing socio-economic status indices: how to use principal components analysis. Health Policy Plan. 2006;21(6):459-68.

27. Lassi ZS, Salam RA, Das JK, Bhutta ZA. Essential interventions for maternal, newborn, and child health : background and methodology. BMC Reprod Health. 2014;11:3-7.

28. Wagstaff A, Van Doorslaer E, Watanabe N: On decomposing the causes of health sector inequalities with an application to malnutrition inequalities in Vietnam: World Bank Washington, DC. . CDC; 2001

29. Orbeta AC Jr. poverty, vulnerability, and family size: evidence from Philippines in: ADB Institute research paper. Tokoyo, Japan: ADB Institute; Sep 2005.

30. Mehari K, Wencheko E. Factors affecting maternal health care services utilization in rural Ethiopia: a study based on the 2011 EDHS data. Ethiop J Health Dev. 2013;27(1):16-24.

31. Regassa N. Antenatal and postnatal care service utilization in southern Ethiopia: a population-based study. Afr Health Sci. 2011;11(3)

32. Van Doorslaer $E$, Wagstaff $A$, Lindelow M. Analyzing health equity using household survey data. Washington, DC: The Word Bank. p. 2008.

33. Gerdtham UG. Equity in health care utilization: further tests based on hurdle models and Swedish micro data. Health economics 1997;6(3):303-19.

34. Wagstaff A. Reply to Guido Erreygers and tom van Ourti's comment on 'the concentration index of a binary outcome revisited'. Health Econ. 2011; 20(10):1166-8

35. Kakwani N, Wagstaff A, Van Doorslaer E. Socioeconomic inequalities in health: measurement, computation, and statistical inference. J Economet. 1997:77(1):87-103.

36. Litchfield JA. Inequality: methods and tools, vol. 4: World Bank; 1999

37. O'Donnell O, O'Neill S, Van Ourti T, Walsh B. Conindex: estimation of concentration indices. The Stata J. 2016;16(1):112.

38. ADePT: a Great Software for Data \& Analytical Reports [http://blogs.worldbank org/growth/adept-great-software-data-analytical-reports]. Accessed 19 July 2017.

39. Graubard BI, Korn EL. Analyzing health surveys for cancer-related objectives. J Natl Cancer Inst. 1999:91(12):1005-16.

40. Mutangadura G, Gauci A, Armah B, Woldemariam E, Ayalew D, Egu B. Health inequities in selected African countries: review of evidence and policy implications. Economic Commission for Africa. 2007;

41. Memirie ST, Verguet S, Norheim OF, Levin C, Johansson KA. Inequalities in utilization of maternal and child health services in Ethiopia: the role of primary health care. BMC Health Serv Res. 2016;16(1):51.

42. Chaka EE. Inequalities in the utilization of maternal health services by key indicators in Ethiopia. J Health, Medicine and Nursing. 2017:39

43. Spangler SA, Barry D, Sibley L. An evaluation of equitable access to a community-based maternal and newborn health program in rural Ethiopia. J Midwifery Women's Health. 2014;59(s1)
44. Yaya S, Bishwajit G, Shah V. Wealth, education and urban-rural inequality and maternal healthcare service usage in Malawi. BMJ global health. 2016;1(2)

45. Dingle A, Powell-Jackson T, Goodman C. A decade of improvements in equity of access to reproductive and maternal health services in Cambodia, 2000-2010. Int J Equity Health. 2013;12(1):51.

46. Navodaya AR, Chauhan BG: Inequality in utilization of maternal health care services among teenage married women in Uttar Pradesh: evidences form NFHS-3. 2014.

47. Hajizadeh M, Alam N, Nandi A. Social inequalities in the utilization of maternal care in Bangladesh: have they widened or narrowed in recent years? Int J Equity Health. 2014;13(1):120.

48. Rahman A, Anwar I, Nisha MK, Alam N, Ahmed S, Begum T. Trends, determinants and inequities of 4+ ANC utilisation in Bangladesh. J Health Popul Nutr. 2017;36(1):2

49. Obiyan $\mathrm{MO}$, Kumar A. Socioeconomic inequalities in the use of maternal health care services in Nigeria: trends between 1990 and 2008. SAGE Open. 2015:5(4):2158244015614070.

50. Mezmur M, Navaneetham K, Letamo G, Bariagaber H. Socioeconomic inequalities in the uptake of maternal healthcare services in Ethiopia. BMC Health Serv Res. 2017;17(1):367.

51. Ethiopia Population [http://worldpopulationreview.com/countries/ethiopiapopulation/. Accessed 16 March 2017].

52. Ethiopia:Analytical summary - Health workforce - AHO [http://www.aho.afro. who.int/profiles_information/index.php/Ethiopia:Analytical_summary_Health_workforce. Accessed 21 March 2017].

53. Gupta A, Kumar P, Dorcas OA. Decomposing the socio-economic inequalities in utilization of full antenatal care in Jharkhand state, India. Int J Population Studies. 2016;2(2)

54. Goland E, Hoa DTP, Målqvist M. Inequity in maternal health care utilization in Vietnam. Int J Equity Health. 2012;11(1):24.

55. Nawal D, Sekher T, Goli S. Decomposing the socioeconomic inequality in utilisation of maternal health-care services in selected Asian and subSaharan African countries. Lancet. 2013:381:S97.

56. A systematic review of inequalities in the use of maternal health care in developing countries: examining the scale of the problem and the importance of context [http://www.who.int/bulletin/volumes/85/10/06035659/en/]. Accessed 10 Feb 2018.

57. Zere $\mathrm{E}$, Tumusiime P, Walker O, Kirigia J, Mwikisa C, Mbeeli T. Inequities in utilization of maternal health interventions in Namibia: implications for progress towards MDG 5 targets. Int J Equity Health. 2010;9(1):16.

58. Mezmur M, Navaneetham K, Letamo G, Bariagaber H. Individual, household and contextual factors associated with skilled delivery care in Ethiopia: evidence from Ethiopian demographic and health surveys. PLoS One. 2017; 12(9):e0184688

59. Chou D, Inoue M, Mathers C, Oestergaard M, Say L, Mills S, Suzuki E, Wilmoth J: Trends in maternal mortality: 1990 to 2008. Estimates developed by Who unicef unfpa and The World Bank. 2010

60. Whitehead $\mathrm{M}$. The concepts and principles of equity and health. Health Promot Int. 1991;6(3):217-28.

61. Leenan $\mathrm{H}$ : Equality and equity in health care. In: WHO/Nuffield Centre for Health Service Studies meeting: 1985; 1985.
Ready to submit your research? Choose BMC and benefit from:

- fast, convenient online submission

- thorough peer review by experienced researchers in your field

- rapid publication on acceptance

- support for research data, including large and complex data types

- gold Open Access which fosters wider collaboration and increased citations

- maximum visibility for your research: over $100 \mathrm{M}$ website views per year

At BMC, research is always in progress.

Learn more biomedcentral.com/submissions 